\title{
Efeito da calagem e da correção dos teores de Ca e Mg do solo sobre o crescimento de mudas de angelim- pedra (Dinizia excelsa Ducke)
}

Juliana dos Santos SENA ${ }^{1}$, Carlos Alberto Franco TUCCI ${ }^{2}$, Hedinaldo Narciso LIMA ${ }^{3}$, Francisco Adilson dos Santos HARA ${ }^{4}$

\begin{abstract}
RESUMO
O conhecimento dos requerimentos nutricionais das espécies e de suas respostas à correçáo do substrato é fundamental para a produçáo de mudas de qualidade. O objetivo deste trabalho foi avaliar o efeito de diferentes tipos de calcário e da correçáo da deficiência de $\mathrm{Ca}$ e $\mathrm{Mg}$ sobre o desenvolvimento de mudas de angelim-pedra (D. excelsa) em casa de vegetação, utilizandose como substrato Latossolo Amarelo. Foram testados três tipos de calcário e fornecimento de $\mathrm{Ca}$ e $\mathrm{Mg}$ por meio de fontes não-corretivas da acidez em três relaçóes $\mathrm{Ca}: \mathrm{Mg}$. Os tratamentos consistiram de $\mathrm{T}_{0}-$ testemunha; $\mathrm{T}_{1}-$ calcário dolomítico; $\mathrm{T}_{2}$ - calcário magnesiano; $\mathrm{T}_{3}$ - calcário calcítico; $\mathrm{T}_{4}$ - Ca e $\mathrm{Mg}$ na relaçáo 3:1; $\mathrm{T}_{5}-\mathrm{Ca}$ e $\mathrm{Mg}$ na relação 9:1; e $\mathrm{T}_{6}-\mathrm{Ca}$ e $\mathrm{Mg}$ na relação 15:1. O delineamento experimental foi de blocos ao acaso, com cinco repetiçôes, totalizando 35 parcelas, cada uma delas com 3 mudas. O substrato foi adubado com doses equivalentes a 100-250-150 e $15 \mathrm{~kg} \mathrm{ha}^{-1} \mathrm{de} \mathrm{N}, \mathrm{P}_{2} \mathrm{O}_{5}, \mathrm{~K}_{2} \mathrm{O}$ e $\mathrm{S}$, respectivamente e com solução de micronutrientes $\left(3 \mathrm{~mL}\right.$ de Chelamix $\mathrm{L}^{-1}$ de água destilada). Foram avaliadas: a altura da planta, o diâmetro do colo, matéria seca da parte aérea, matéria seca da raiz, matéria seca total, relaçáo raiz/parte aérea e conteúdos de nutrientes da parte aérea das plantas. De modo geral, a aplicaçáo de calcário favoreceu o crescimento de $D$. excelsa, sendo os melhores resultados obtidos com a aplicação de calcário magnesiano na relação 9:1.
\end{abstract}

PALAVRAS-CHAVE: produção de mudas, calagem, Dinizia excelsa.

\section{Liming effect and correction of the $\mathrm{Ca}$ and $\mathrm{Mg}$ contents of the soil on angelim-pedra (Dinizia excelsa Ducke) seedling growth}

\begin{abstract}
Knowledge of nutritional requirements of the species and their answers to-correcting the substratum is fundamental for the production of quality seedlings. In this work, we aimed to evaluate the effect of different limestone types and the correction of $\mathrm{Ca}$ and $\mathrm{Mg}$ deficiency on the development of angelim-pedra (Dinizia excelsa) seedlings, under glasshouse conditions. We tested three types of limestone and three types of Ca:Mg provisioning-on a Yellow Oxisol (with non-corrective acidity sources): $\mathrm{T}_{0}$ - control; $\mathrm{T}_{1}$ - soil limed with dolomitic lime (3:1); $\mathrm{T}_{2}$ - soil limed with-magnesium lime (9:1); $\mathrm{T}_{3}$ - soil limed with calcitic lime (15:1); $\mathrm{T}_{4}$ - a mixture of calcium and magnesium, in the 3:1 proportion; $\mathrm{T}_{5}$ - calcium and magnesium, in the 9:1 proportion; and $\mathrm{T}_{6}$ - calcium and magnesium, in the 15:1 proportion. The experimental design was of random blocks, with 5 repetitions, totaling 35 parcels with 3 seedlings each. The substratum was fertilized with doses equivalent to 100-250-150 and $15 \mathrm{k} \mathrm{ha}^{-1}$ of N, $\mathrm{P}_{2} \mathrm{O}_{5}, \mathrm{~K}_{2} \mathrm{O}$ and $\mathrm{S}$ respectively, and-micronutrient solution ( $3 \mathrm{~mL}$ of Chelamix $\mathrm{L}^{-1}$ of distilled water). We evaluated the height, diameter of the stem, dry biomass of the aerial part, dry biomass of the root, dry biomass of the drought, aerial part/root relation and nutrient contents of the aerial part of the plants. The limestone application affected positively the height, the diameter, the matter total biomass and the absorption of Ca of the D. excelsa seedlings $(\mathrm{p}<0,05)$. The application of magnesium lime with Ca:Mg 9:1 proportion showed the best results.
\end{abstract}

KEY WORDS: seedling formation, liming, Dinizia excelsa.

\footnotetext{
1 Instituto Brasileiro do Meio Ambiente, E-mail: sena_juliana@yahoo.com.br

2 Universidade Federal do Amazonas - UFAM, E-mail: ctucci@ufam.edu.br

${ }^{3}$ Universidade Federal do Amazonas - UFAM, E-mail: hedinaldo@ufam.edu.br

${ }^{4}$ Universidade Federal do Amazonas - UFAM, E-mail: fhara@ufam.edu.br
} 


\section{INTRODUÇÃO}

A regiáo amazônica possui grandes áreas desmatadas, que foram abertas para implantação de pastagens, porém a baixa fertilidade dos solos ocasiona a formação de pastagens de baixa produtividade que logo são abandonadas, fazendo com que surjam grandes áreas recobertas por vegetação secundária (Uhl et al. 1988). Uma das alternativas viáveis para diminuir as pressôes de desmatamento sobre as áreas de floresta primária é a utilização de áreas ocupadas por vegetação secundária, para a instalação de atividades silviculturais (Gascon e Moutinho 1998), em que pequenos agricultores poderão realizar plantios de enriquecimento, com espécies florestais de interesse econômico ou implantar sistemas agroflorestais (Fearnside 1998).

O sucesso do plantio das espécies arbóreas depende em grande parte da qualidade das mudas, além do manejo inicial das espécies cultivadas. Destaca-se que na produção de mudas de qualidade, as características do substrato são fundamentais para o crescimento e o estabelecimento das mudas após o plantio.

Entre os muitos materiais utilizados na formação do substrato, o material dos horizontes subsuperficiais do solo são muitos frequentemente utilizados por sua abundância e menor custo em comparaçáo aos outros materiais. Além disso, de acordo com Paiva e Gomes (2000), esse material é, geralmente, isento de sementes de plantas indesejáveis e microorganismos patogênicos, sendo o mais indicado para a composição do substrato.

A maioria dos solos de terra firme da Amazônia brasileira é ácida e pobres em nutrientes, limitando o crescimento de várias espécies vegetais. Essas limitaçôes podem ser corrigidas por meio do uso de corretivos, em geral calcários, e fertilizantes químicos ou orgânicos. A calagem, além de reduzir a acidez do solo, aumenta a disponibilidade de $\mathrm{Ca}$ e $\mathrm{Mg}$ e reduz a concentração do $\mathrm{Al}$ tóxico.

Entre as várias características dos corretivos, destaca-se a relaçáo $\mathrm{Ca}: \mathrm{Mg}$, que pode alterar a resposta das espécies cultivadas (Quaggio 2000; Venturini et al. 2000; Veloso et al. 2001; Gomes et al. 2002). De acordo com Lima et al. (1981) apud Venturini et al. (2000), se o equilíbrio Ca:Mg não é adequado, há condiçôes para a deficiência induzida de um dos nutrientes, como conseqüência de antagonismos na sua absorção.

Considerando a importância da fase de produçáo de mudas para o cultivo de espécies florestais, o objetivo deste trabalho foi avaliar as respostas de angelim-pedra (D. excelsa Ducke), à aplicação de calcário como corretivo do substrato, bem como identificar os teores de $\mathrm{Ca}$ e $\mathrm{Mg}$ apropriados para o melhor desenvolvimento das plantas.

\section{MATERIAL E MÉTODOS}

O experimento foi conduzido na casa de vegetação, no Campus Universitário da Universidade Federal do Amazonas (UFAM), em Manaus - AM, no período de fevereiro de 2007 a janeiro de 2008.

O material utilizado como substrato foi coletado na profundidade de $20-40 \mathrm{~cm}$ de um Latossolo Amarelo, no mini-campus da UFAM. Após a coleta, o solo foi destorroado e colocado para secar ao ar livre, peneirado em malha de 4 $\mathrm{mm}$ de abertura.

Após o preparo, o substrato foi adicionado em vasos plásticos com capacidade para $12 \mathrm{~kg}$ de solo. Os tratamentos foram aplicados individualmente em cada vaso e em seguida o substrato foi homogeneizado, com auxílio de uma betoneira. Após a homogeneizaçáo, o substrato foi colocado novamente nos vasos e incubados por 30 dias, mantendo-se a umidade dos vasos a $30 \%$ da capacidade de campo.

Os tratamentos consistiram na aplicação de três tipos de calcário e de três compostos químicos não-corretivos de acidez, contendo $\mathrm{Ca}$ e $\mathrm{Mg}$ na proporção correspondente a cada tipo de calcário, além do tratamento testemunha, que consistiu no uso do substrato sem qualquer adição, a saber:

$\mathrm{T}_{0}$ - substrato sem calagem ou adição de Ca e Mg;

$\mathrm{T}_{1}$ - substrato corrigido com calcário dolomítico, relação Ca:Mg 3:1;

$\mathrm{T}_{2}$ - substrato corrigido com calcário magnesiano, relação Ca:Mg 9:1;

$\mathrm{T}_{3}$ - substrato corrigido com calcário calcítico, relação Ca:Mg 15:1;

$\mathrm{T}_{4}$ - aplicação de Ca e $\mathrm{Mg}$ na relação Ca: $\mathrm{Mg}$ de 3:1;

$\mathrm{T}_{5}$ - aplicação de Ca e Mg na relaçáo Ca:Mg de 9:1;

$\mathrm{T}_{6}$ - aplicação de Ca e Mg na relação Ca:Mg de 15:1.

Nos tratamentos $T_{1}, T_{2}$ e $T_{3}$, foi utilizada uma mistura de $\mathrm{CaCO}_{3}$ (р.a.) e $\left(\mathrm{MgCO}_{3}\right)_{4} \mathrm{Mg}(\mathrm{OH})_{2} 5 \mathrm{H}_{2} \mathrm{O}$ (p.a.), em proporçóes equivalentes às relaçóes $\mathrm{Ca} e \mathrm{Mg}$ dos diferentes tipos de corretivos utilizados com poder relativo de neutralizaçáo total (PRNT) igual a 100\%.

Como fonte de $\mathrm{Ca}$ e $\mathrm{Mg}$ não-corretiva da acidez, aplicada nos tratamentos $\mathrm{T}_{4}, \mathrm{~T}_{5} \mathrm{e} \mathrm{T}_{6}$, utilizou-se $\mathrm{CaCl}_{2} \cdot 2 \mathrm{H}_{2} \mathrm{O}$ e $\mathrm{MgCl}_{2} \cdot 6 \mathrm{H}_{2} \mathrm{O}$, aplicadas em proporçôes equivalentes as relaçóes Ca e Mg utilizadas nos tratamentos $\mathrm{T}_{1}, \mathrm{~T}_{2} \mathrm{e} \mathrm{T}_{3}$. Independente da fonte, os produtos utilizados corresponderam, em quantidade de corretivo, à dose equivalente de $2 \mathrm{tha}^{-1}$.

Depois do período de incubação, o substrato foi novamente seco, destorroado e peneirado. Daí procedeu-se a fertilizaçáa do substrato com a utilizaçáo de ácido fosfórico $\left(\mathrm{H}_{3} \mathrm{PO}_{4}\right)$, cloreto de potássio $(\mathrm{KCl})$ e sulfato de potássio $\left(\mathrm{K}_{2} \mathrm{SO}_{4}\right)$, em doses equivalentes a 250,150 e $15 \mathrm{~kg} \mathrm{ha}^{-1} \mathrm{de}$ 
$\mathrm{P}_{2} \mathrm{O}_{5}, \mathrm{~K}_{2} \mathrm{O}$ e $\mathrm{S}$. As fontes de $\mathrm{P}, \mathrm{Ke} \mathrm{S}$ foram aplicadas na forma de solução e misturadas ao substrato.

Após a secagem, o substrato foi peneirado, homogeneizado e transferido para os citropotes, com capacidade para $3,2 \mathrm{~kg}$, devidamente identificados com os respectivos tratamentos. Foi feita uma fertilização com $\mathrm{N}$ em cobertura, utilizando-se uréia $\left(\mathrm{CO}\left(\mathrm{NH}_{3}\right)_{2}\right)$, em dose equivalente a $100 \mathrm{~kg} \mathrm{ha}^{-1} \mathrm{de} \mathrm{N}$, aplicada na forma de solução em três parcelas, 40,30 e 30 $\mathrm{kg}$ de $\mathrm{N} \mathrm{ha}^{-1}$, aplicadas 15, 45 e 60 dias depois da repicagem das plântulas.

Um fertilizante à base de micronutrientes quelatizados, denominado Chelamix ${ }^{\oplus}$, foi aplicado em doses correspondestes a $0,5 \%$ de B, $0,2 \%$ de $\mathrm{Cu}, 0,3 \%$ de $\mathrm{Fe}, 0,2 \%$ de $\mathrm{Mn}, 0,5 \%$ de Mo e 2,4\% de $\mathrm{Zn}$ vaso ${ }^{-1}$, na forma de solução $(3 \mathrm{~mL}$ de Chelamix $\mathrm{L}^{-1}$ de água destilada), sendo $50 \mathrm{~mL}$ de solução vaso $^{-1}$ a cada 15 dias após a repicagem em todos os ensaios, de acordo com Silva Junior (2006).

Antes da aplicação dos tratamentos, uma amostra composta do substrato foi retirada para a realizaçáo de análises físicas e químicas, no Laboratório de Fertilidade de Solo e Análise de Planta da FCA/UFAM, conforme metodologia da EMBRAPA (1997). Nas análises físicas foram obtidos os valores de argila total, areia e silte. Nas análises químicas do solo foram obtidos os valores de macronutrientes (P, K, Ca e $\mathrm{Mg}$ ), $\mathrm{pH}$ em $\mathrm{H}_{2} \mathrm{O}$, matéria orgânica (MO), acidez trocável e potencial. A partir desses dados foram calculados os valores de soma de bases $(\mathrm{S})$, capacidade de troca catiônica a pH 7,0 (T) e a CTC efetiva $(t)$, saturação por bases $(V \%)$ e a saturação por alumínio (m) (Tabela 1). Para verificar possíveis alteraçôes nesses atributos, foi realizada outra análise química após a calagem e fertilização do substrato (Tabela 2).

Durante o experimento, a irrigação foi realizada diariamente, mantendo a umidade das unidades experimentais a 30\% da capacidade de campo, obtida pelo método de pesagem.

As sementes de angelim-pedra, coletadas na Reserva Florestal Adolpho Ducke, do Instituto Nacional de Pesquisas da Amazônia (INPA), foram inicialmente armazenadas em câmara fria a $10^{\circ} \mathrm{C}$.

As sementes foram imersas em ácido sulfúrico (98\%) por 20 minutos e, posteriormente, lavadas em água corrente (Vastano Júnior et al 1983) e, em seguida, foram colocadas para germinar em sementeiras plásticas retangulares $(42 \times 27$ $\mathrm{x} 8 \mathrm{~cm}$ ), usando-se como substrato areia lavada. A repicagem das plântulas foi realizada 31 dias após a emergência.

Após 152 dias em casa de vegetação, foram medidos a altura das mudas, utilizando-se régua graduada, desde o nível do substrato até a gema apical, e o diâmetro do colo a $1 \mathrm{~cm}$ da superfície do solo, empregando-se paquímetro digital. As mediçôes foram feitas em apenas duas mudas por parcela, devido à mortalidade de algumas plantas, totalizando 70 mudas.

Após as mediçôes, realizou-se a colheita do material vegetal, separando-se em parte aérea e raiz, as quais foram acondicionadas em sacos de papel e levadas à estufa de ventilação forçada, à temperatura de $60^{\circ} \mathrm{C}$, até a obtenção de peso constante. $\mathrm{O}$ material seco foi, em seguida, pesado em balança analítica de precisão, obtendo-se o peso da matéria seca da parte aérea (MSPA), o peso da matéria seca da raiz (MSR) e, por somatória, o peso da matéria seca total (MST). A relação raiz/parte aérea (RRPA) foi obtida pela relaçâo entre o peso da matéria seca da raiz e o peso da matéria seca da parte aérea.

O material vegetal da parte aérea foi submetido à análise, conforme Malavolta et al. (1997) para determinação dos teores de N, P, K, Ca, Mg, B, Cu, Fe, Mn e Zn. A partir desses resultados, determinou-se o conteúdo de nutrientes na parte aérea das mudas, multiplicando-se o teor de cada elemento pela massa seca da parte aérea.

O crescimento relativo (CR) em altura, diâmetro, MSPA, MSR e MST foram estimados pela razão entre as características de crescimento medidas nos tratamentos $\mathrm{T}_{1} \mathrm{a}_{6}$ e os valores da testemunha $\left(\mathrm{T}_{0}\right)$, multiplicado por $100 \%$.

Os dados foram submetidos a análises de variância, utilizando-se o programa SAEG 9.1. As médias foram comparadas pelo teste de Scott-Knott a 5\% de probabilidade.

\section{RESULTADOS E DISCUSSÃO}

\section{ANÁLISE QUIIMICA DO SUBSTRATO}

Os resultados apresentados na Tabela 1 mostram que o solo utilizado como substrato apresentou, inicialmente, acidez elevada, baixa saturação por bases, baixa CTC efetiva e baixos teores de $\mathrm{P}$ disponível, $\mathrm{Ca}^{2+}$ e $\mathrm{Mg}^{2+}$ trocáveis. $\mathrm{O}$ teor de $\mathrm{K}$ disponível, a acidez trocável e potencial foram considerados medianos (CFSEMG 1999).

Tabela 1 - Características químicas e físicas do solo utilizado como substrato, antes da aplicação dos tratamentos.

\begin{tabular}{|c|c|c|c|c|c|c|c|}
\hline $\mathrm{pH}$ & K & $P$ & $\mathrm{Al}^{3+}$ & $\mathrm{Ca}^{2+}$ & $\mathrm{Mg}^{2+}$ & $\mathrm{H}+\mathrm{Al}$ & MO \\
\hline$\left(\mathrm{H}_{2} \mathrm{O}\right)$ & \multicolumn{2}{|c|}{$\mathrm{mg} \mathrm{kg}^{-1}$} & \multicolumn{4}{|c|}{$\mathrm{cmol}_{(\mathrm{c})} \mathrm{kg}^{-1}$} & $\mathrm{~g} \mathrm{~kg}^{-1}$ \\
\hline 4,46 & 44 & 3 & 0,68 & 0,7 & 0,4 & 4,19 & 20,22 \\
\hline \multirow[t]{2}{*}{ Areia } & Silte & Argila & S & $\mathrm{T}$ & $\mathrm{t}$ & V & $\mathrm{m}$ \\
\hline & $\%$ & & \multicolumn{3}{|c|}{$\mathrm{cmol}_{(\mathrm{c})} \mathrm{kg}^{-1}$} & \multicolumn{2}{|c|}{$\%$} \\
\hline 12,56 & 1,21 & 86,23 & 1,24 & 5,42 & 1,91 & 22,82 & 35,29 \\
\hline
\end{tabular}

S - soma de bases; $\mathrm{T}$ - capacidade de troca catiônica a pH 7,0; $\mathrm{t}$ - CTC efetiva; V\% saturação por bases; $m$ - saturação por alumínio. 
A aplicaçáo dos corretivos e fertilizantes referentes aos tratamentos deste estudo promoveu alteraçóes na composição química do substrato de cultivo em todas as características avaliadas (Tabela 2). Houve uma reduçáo na acidez do substrato em todos os tratamentos, no entanto, observou-se que nos tratamentos onde houve a prática da calagem foram encontrados os maiores valores de $\mathrm{pH}$, além dos menores teores de $\mathrm{Al}^{3+}$, acidez potencial $(\mathrm{H}+\mathrm{Al})$ e saturação de alumínio.

Tabela 2 - Características químicas do substrato 30 dias após a aplicação dos tratamentos.

\begin{tabular}{|c|c|c|c|c|c|c|}
\hline \multirow{2}{*}{ Tratamentos } & $\mathrm{pH}$ & K & $P$ & $\mathrm{Ca}^{2+}$ & $\mathrm{Mg}^{2+}$ & $S$ \\
\hline & $\mathrm{H}_{2} \mathrm{O}$ & \multicolumn{2}{|c|}{$\mathrm{mg} \mathrm{kg}^{-1}$} & \multicolumn{3}{|c|}{$\mathrm{cmol}_{(\mathrm{c})} \mathrm{kg}^{-1}$} \\
\hline $\mathrm{T}_{0}$ & $4,47 d$ & $79,9 a$ & $33,9 a$ & $0,54 d$ & $0,23 b$ & 0,97 \\
\hline $\mathrm{T}_{1}$ & $5,85 b$ & $78,5 a$ & $30,0 \mathrm{a}$ & $1,72 \mathrm{a}$ & $0,45 a$ & 2,37 \\
\hline $\mathrm{T}_{2}$ & $6,12 \mathrm{a}$ & $69,2 b$ & $33,8 \mathrm{a}$ & $1,74 a$ & $0,50 a$ & 2,42 \\
\hline $\mathrm{T}_{3}$ & $6,20 \mathrm{a}$ & $78,0 \mathrm{a}$ & $25,7 a$ & $1,71 \mathrm{a}$ & $0,26 b$ & 2,17 \\
\hline $\mathrm{T}_{4}$ & $5,76 b$ & $54,7 \mathrm{c}$ & $36,3 a$ & $0,82 \mathrm{c}$ & $0,33 b$ & 1,29 \\
\hline $\mathrm{T}_{5}$ & $5,71 b$ & $59,5 c$ & $36,4 a$ & $0,89 c$ & $0,44 a$ & 1,49 \\
\hline $\mathrm{T}_{6}$ & $5,51 \mathrm{c}$ & $66,3 d$ & $34,0 \mathrm{a}$ & $1,16 \mathrm{~b}$ & $0,25 b$ & 1,57 \\
\hline \multirow{2}{*}{ Tratamentos } & $\mathrm{Al}^{3+}$ & $\mathrm{H}+\mathrm{Al}$ & $\mathrm{T}$ & $\mathrm{t}$ & V & $\mathrm{m}$ \\
\hline & \multicolumn{4}{|c|}{$\mathrm{cmol}_{(\mathrm{c})} \mathrm{kg}^{-1}$} & \multicolumn{2}{|c|}{$\%$} \\
\hline $\mathrm{T}_{0}$ & $0,19 a$ & $3,9 a$ & 4,9 & 1,16 & 19,83 & 16,19 \\
\hline $\mathrm{T}_{1}$ & $0,07 b$ & $2,5 c$ & 4,89 & 2,44 & 48,59 & 2,81 \\
\hline $\mathrm{T}_{2}$ & $0,05 b$ & $2,3 c$ & 4,76 & 2,47 & 50,79 & 2,03 \\
\hline $\mathrm{T}_{3}$ & $0,05 b$ & $3,1 b$ & 5,31 & 2,22 & 40,95 & 2,25 \\
\hline $\mathrm{T}_{4}$ & $0,12 \mathrm{a}$ & $3,4 b$ & 4,66 & 1,41 & 27,77 & 8,58 \\
\hline $\mathrm{T}_{5}$ & $0,09 b$ & $3,4 b$ & 4,92 & 1,58 & 30,18 & 5,91 \\
\hline $\mathrm{T}_{6}$ & $0,18 \mathrm{a}$ & $4,2 \mathrm{a}$ & 5,75 & 1,75 & 27,35 & 10,27 \\
\hline
\end{tabular}

Letras distintas na mesma coluna diferem entre si pelo teste de Scott-Knott, a 5\% de probabilidade. T0 - testemunha: T1 - calcário dolomítico: T2 - calcário magnesiano; T3 - calcário calcítico; T4 - relação Ca:Mg de 3:1; T5 - relação Ca:Mg de 9:1; e T6 - relação Ca:Mg de 15:1; S - soma de bases; T - capacidade de troca catiônica a pH 7,0; $t$ - CTC efetiva; V\% - saturação por bases; $m$ saturação por alumínio.

A prática da calagem em conjunto com a adubação

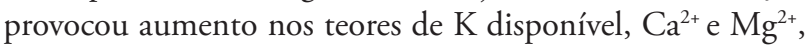
na saturação por bases e na CTC efetiva do substrato. Nos tratamentos onde houve a adição de $\mathrm{Ca}$ e $\mathrm{Mg}\left(\mathrm{T}_{4}, \mathrm{~T}_{5}\right.$ e $\left.\mathrm{T}_{6}\right)$ e testemunha $\left(\mathrm{T}_{0}\right)$, observou-se os maiores teores de $\mathrm{Al}^{3+}$, acidez potencial $(\mathrm{H}+\mathrm{Al})$ e saturaçáo de alumínio $(\mathrm{m} \%)$, além dos menores valores de saturação por bases e CTC efetiva. A toxidez de certos elementos presentes em concentraçôes excessivas na soluçáo do solo, como o Al, é uma das principais causas da baixa capacidade produtiva dos solos ácidos (Furtini Neto et al. 1999). O alumínio em altas concentraçóes resulta em toxidez, inibindo o crescimento das plantas, refletindo principalmente em menor desenvolvimento do sistema radicular (Rossiello e Jacob Neto, 2006; Zonta et al. 2006).

A adição de $\mathrm{Ca}$ e $\mathrm{Mg}$, proveniente dos calcários ou das fontes náo corretivas da acidez, promoveram o aumento

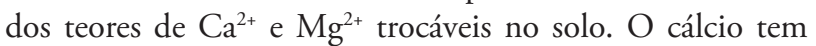
importante papel na absorção iônica, particularmente na correção do efeito desfavorável da concentração hidrogeniônica excessiva, sendo essencial para que tal efeito náo diminua a absorção de nutrientes (Vitti et al. 2006). A presença de $\mathrm{Al}^{3+}$ em concentraçóes elevadas no solo, além de ser considerado tóxico para as plantas, interfere na disponibilidade de nutrientes, principalmente do P (Barros 2001; Rossiello e Jacob Neto 2006).

\section{ABSORÇÃO DE NUTRIENTES}

A análise de variância mostrou efeito significativo dos tratamentos sobre o conteúdo de $\mathrm{N}(\mathrm{F}=2,908 ; \mathrm{p}<0,05)$. Verificou-se que as mudas tratadas com calcário magnesiano $\left(\mathrm{T}_{2}\right)$, calcário calcítico $\left(\mathrm{T}_{3}\right)$ e relaçáo Ca:Mg de 9:1 $\left(\mathrm{T}_{5}\right)$ foram as que apresentaram maiores conteúdos de $\mathrm{N}$ (Tabela 3). Isso indica que a absorçáo de $\mathrm{N}$ pode ser afetada pelos teores de $\mathrm{Ca}^{2+}$ e $\mathrm{Mg}^{2+}$ no solo. Os maiores valores de $\mathrm{N}$ na planta foram observados quando a relaçấo Ca:Mg aplicada ao solo foi de 9:1 $\left(\mathrm{T}_{2} \mathrm{e} \mathrm{T}_{5}\right)$. O conteúdo de $\mathrm{N}$ nas mudas produzidas com calcário calcítico $\left(\mathrm{T}_{3}\right)$ foi diferente do encontrado nas mudas produzidas com a adição de Ca:Mg na relação 15:1 $\left(\mathrm{T}_{6}\right)$, mostrando a importância de uma adequada relação Ca:Mg no substrato de cultivo. Semelhantemente, Silva (2004), estudando o efeito da calagem na produçáo de mudas de sumaúma (Ceiba pentandra L. Gaertn), verificou que o conteúdo de $\mathrm{N}$ na parte aérea e raiz foi afetado positivamente pela calagem, possivelmente devido à elevaçáo do $\mathrm{pH}$ do solo que provoca aumento na decomposição da matéria orgânica e, conseqüentemente, a maior mineralizaçáo do nitrogênio, após a calagem.

A calagem, bem como a correção dos teores $\mathrm{da} \mathrm{Ca}^{2+} \mathrm{e}$ $\mathrm{Mg}^{2+}$ no solo, náo influenciou a absorçáo de $\mathrm{P}$ nas mudas de angelim-pedra (Tabela 3). Da mesma forma, Ulhôa (1997), verificou que o conteúdo de $P$ na parte aérea das mudas de baru (Dipterys alata $\mathrm{Vog}$ ), não apresentou nenhuma correlação com a calagem. Entretanto, Silva (2004) observou que a absorção de $\mathrm{P}$, na parte aérea e raiz de mudas de sumaúma (C. pentandra), foi influenciada positivamente pela calagem.

Houve efeito significativo tanto da calagem quanto da correçáo de Ca e Mg no substrato de cultivo sobre o conteúdo de $K$ nas plantas $(F=2,915 ; p<0,05$; Tabela 3). A absorção deste nutriente foi superior nos tratamentos com calcário magnesiano $\left(\mathrm{T}_{2}\right)$, calcário calcítico $\left(\mathrm{T}_{3}\right)$ e aplicação de Ca e Mg na relação 9:1 $\left(T_{5}\right)$, os demais tratamentos $\left(T_{1}, T_{4}, T_{6}\right)$ foram estatisticamente iguais à testemunha $\left(\mathrm{T}_{0}\right)$. Resultados semelhantes a estes foram observados por Silva (2004), 
ao estudar o efeito da calagem na produçáo de mudas de sumaúma ( $C$. pentandra), em que o conteúdo de $\mathrm{K}$ na parte aérea e raiz das mudas sofreram efeito positivo da calagem.

A estreita relação Ca: $\mathrm{Mg}$ no calcário dolomítico $\left(\mathrm{T}_{1}\right)$, bem como a adição de Ca e Mg na relaçáo 3:1 ( $\left.\mathrm{T}_{4}\right)$, aparentemente, estaria prejudicando a absorção do K. Ulhôa (1997), observou que o conteúdo de $\mathrm{K}$ nas mudas de baru ( $D$. alata) apresentou um decréscimo com o aumento das doses de $\mathrm{P}$ e calcário. De acordo com este autor, possivelmente as concentraçôes de $\mathrm{Ca}$ e $\mathrm{Mg}$ no solo poderiam estar inibindo a absorção de $\mathrm{K}$ pela planta.

Em relação ao conteúdo de Ca na parte aérea das mudas, verificou-se que houve diferença significativa entre os diferentes tratamentos aplicados $(\mathrm{F}=3,50 ; \mathrm{p}<0,05)$. O conteúdo de $\mathrm{Ca}$ foi estatisticamente igual entre os tratamentos $\mathrm{T}_{1}, \mathrm{~T}_{2}, \mathrm{~T}_{3} \mathrm{e} \mathrm{T}_{5}$, sendo superiores aos dos tratamentos $\mathrm{T}_{4}, \mathrm{~T}_{6}$ e $\mathrm{T}_{0}$, também estatisticamente iguais (Tabela 3). A absorção de Ca foi afetada positivamente pela calagem. De modo semelhante, a calagem teve efeito positivo sobre o conteúdo de Ca na parte aérea e raiz em mudas de sumaúma (C.pentandra), em que o aumento no teor de $\mathrm{Ca}^{2+}$ e $\mathrm{Mg}^{2+}$ no solo têm como conseqüência um maior conteúdo desses nutrientes na planta, não observando antagonismo entre a absorção de Ca e Mg (Silva 2004). Outros trabalhos também apontam resultados semelhantes a estes (Ulhôa 1997; Barros 2001; Silva et al. 2007).

Não houve diferença significativa no conteúdo de Ca entre os diferentes tipos de calcários. No entanto, quando aplicado Ca e Mg no solo, por fontes náo corretivas da acidez, observase diferença significativa na absorção de $\mathrm{Ca}$ entre as diferentes relaçóes Ca:Mg. Nas relaçôes 3:1 e 15:1, as mudas de angelimpedra produzidas apresentaram os menores conteúdos de $\mathrm{N}$, $\mathrm{P}, \mathrm{K}, \mathrm{Ca}, \mathrm{Mg}$ e $\mathrm{S}$ na parte aérea, embora para os dois últimos nutrientes a diferença não seja significativa (Tabela 3).

Em relação à absorção de $\mathrm{Mg}$, verificou-se que náo houve efeito dos tratamentos sobre o conteúdo deste nutriente na parte aérea das mudas de angelim-pedra. A absorçáo de $\mathrm{Mg}$ não foi influenciada pela correção da acidez nem pela adição de $\mathrm{Ca}$ e $\mathrm{Mg}$ por fontes náo corretivas da acidez (Tabela 3). Isso sugere que a espécie pode não ser táo exigente neste nutriente, ou que a quantidade $\mathrm{Mg}^{2+}$ presente no solo foi suficiente para suprir as necessidades das plantas. Ao testar o efeito de doses de calcário sobre a absorçâo de nutrientes pela parte aérea e raiz de mudas de sumaúma ( $C$. pentandra), Silva (2004) verificou que o maior estoque de $\mathrm{Ca}$ e de $\mathrm{Mg}$ foram encontrados sob a aplicação da maior dose utilizada $(5 \mathrm{t}$ ha $\left.{ }^{1}\right)$. Resultados semelhantes a estes também foram observados por Ulhôa (1997), estudando o efeito da adubação fosfatada e calagem na produçáo de mudas de baru (D. alata).

$O$ conteúdo de $S$ na parte aérea das mudas foi estatisticamente igual em todos os tratamentos (Tabela 4). A aplicação de calcário náo afetou a absorção deste nutriente
Tabela 3 - Conteúdo de macronutrientes na parte aérea das mudas de angelim-pedra produzidas em casa de vegetação por um período de 152 dias.

\begin{tabular}{lcccccc}
\hline \multirow{2}{*}{ Tratamentos } & $\mathrm{N}$ & $\mathrm{P}$ & $\mathrm{K}$ & $\mathrm{Ca}$ & $\mathrm{Mg}$ & $\mathrm{S}$ \\
\cline { 2 - 7 } & \multicolumn{7}{c}{ (g citropote $^{-1}$ ) } \\
\hline $\mathrm{T}_{0}$ & $2,30 \mathrm{~b}$ & $0,07 \mathrm{a}$ & $0,97 \mathrm{~b}$ & $0,59 \mathrm{~b}$ & $0,09 \mathrm{a}$ & $0,15 \mathrm{a}$ \\
$\mathrm{T}_{1}$ & $2,81 \mathrm{~b}$ & $0,11 \mathrm{a}$ & $1,26 \mathrm{~b}$ & $1,20 \mathrm{a}$ & $0,15 \mathrm{a}$ & $0,20 \mathrm{a}$ \\
$\mathrm{T}_{2}$ & $4,21 \mathrm{a}$ & $0,15 \mathrm{a}$ & $1,85 \mathrm{a}$ & $1,58 \mathrm{a}$ & $0,15 \mathrm{a}$ & $0,25 \mathrm{a}$ \\
$\mathrm{T}_{3}$ & $3,64 \mathrm{a}$ & $0,17 \mathrm{a}$ & $1,60 \mathrm{a}$ & $1,50 \mathrm{a}$ & $0,12 \mathrm{a}$ & $0,21 \mathrm{a}$ \\
$\mathrm{T}_{4}$ & $3,12 \mathrm{~b}$ & $0,13 \mathrm{a}$ & $1,32 \mathrm{~b}$ & $0,94 \mathrm{~b}$ & $0,14 \mathrm{a}$ & $0,20 \mathrm{a}$ \\
$\mathrm{T}_{5}$ & $4,46 \mathrm{a}$ & $0,21 \mathrm{a}$ & $1,85 \mathrm{a}$ & $1,24 \mathrm{a}$ & $0,16 \mathrm{a}$ & $0,26 \mathrm{a}$ \\
$\mathrm{T}_{6}$ & $2,25 \mathrm{~b}$ & $0,07 \mathrm{a}$ & $0,98 \mathrm{~b}$ & $0,59 \mathrm{~b}$ & $0,08 \mathrm{a}$ & $0,14 \mathrm{a}$ \\
\hline
\end{tabular}

Letras distintas na mesma coluna diferem entre si pelo teste de Scott-Knott, ao nível de $5 \%$ de probabilidade.

pelas mudas de angelim-pedra, assim como a correção dos teores da $\mathrm{Ca}$ e $\mathrm{Mg}$ no solo, indicando que se o substrato de cultivo estiver com adequado teor de $S$, a absorção deste nutriente não será afetada pela acidez do substrato ou pela deficiência de $\mathrm{Ca}$ e $\mathrm{Mg}$.

Não houve efeito significativo dos diferentes tratamentos sobre o conteúdo de B, Mn e Zn na parte aérea das mudas de angelim-pedra (Tabela 4). Entretanto, verificou-se que houve efeito dos tratamentos sobre o conteúdo de $\mathrm{Cu}(\mathrm{F}=$ 5,116; $\mathrm{p}<0,05)$ na parte aérea das mudas de angelim-pedra. As mudas tratadas com Ca e $\mathrm{Mg}$ na relação 9:1 $\left(\mathrm{T}_{5}\right)$ foram as que apresentaram maior absorçáo de $\mathrm{Cu}$. A calagem $\left(\mathrm{T}_{1}, \mathrm{~T}_{2}, \mathrm{~T}_{3}\right)$ não afetou a absorçáo deste nutriente, sendo estatisticamente igual aos demais tratamentos $\left(\mathrm{T}_{4}, \mathrm{~T}_{6} \mathrm{e} \mathrm{T}_{0}\right)$. Semelhantemente, Silva (2004), constatou que a calagem promove a redução no conteúdo de $\mathrm{Cu}$ na parte aérea e raiz das mudas de sumaúma (C. pentandra).

Em relação ao conteúdo de Fe na parte aérea das mudas de angelim-pedra, verificou-se que houve diferença significativa $(\mathrm{F}=4,158 ; \mathrm{p}<0,05)$ entre os tratamentos (Tabela 4). Verificouse que as mudas tratadas com calcário dolomítico $\left(\mathrm{T}_{1}\right)$, calcário magnesiano $\left(T_{2}\right)$ e aplicação de Ca e Mg na relação 9:1 $\left(T_{5}\right)$ foram as que apresentaram maior absorção de Fe. As mudas tratadas com calcário calcítico $\left(\mathrm{T}_{1}\right)$ e aplicaçáo de $\mathrm{Ca}$ e $\mathrm{Mg}$ nas relaçóes 3:1 $\left(\mathrm{T}_{4}\right)$ e 15:1 $\left(\mathrm{T}_{6}\right)$, apresentaram os menores conteúdos de $\mathrm{Fe}$, sendo estatisticamente iguais a testemunha $\left(\mathrm{T}_{0}\right)$. O conteúdo de Fe na parte aérea e raiz de mudas de sumaúma ( $C$. pentandra) também foi afetado positivamente pela calagem (Silva 2004).

De acordo com os conteúdos de nutrientes na matéria seca da parte aérea observa-se a seguinte ordem decrescente de absorção: $\mathrm{N}>\mathrm{K}>\mathrm{Ca}>\mathrm{S}>\mathrm{Mg}=\mathrm{P}>\mathrm{Fe}>\mathrm{Mn}>\mathrm{Zn}>\mathrm{B}>\mathrm{Cu}$. 
Tabela 4 - Conteúdo de micronutrientes na parte aérea das mudas de angelimpedra produzidas em casa de vegetação por um período de 152 dias.

\begin{tabular}{lccccc}
\hline \multirow{2}{*}{ Tratamentos } & B & Cu & $F e$ & $M n$ & Zn \\
\cline { 2 - 6 } & $0,0040 \mathrm{a}$ & $0,0006 \mathrm{~b}$ & $0,083 \mathrm{~b}$ & $0,009 \mathrm{a}$ & $0,008 \mathrm{a}$ \\
\hline $\mathrm{T}_{0}$ & $0,0051 \mathrm{a}$ & $0,0005 \mathrm{~b}$ & $0,123 \mathrm{a}$ & $0,013 \mathrm{a}$ & $0,010 \mathrm{a}$ \\
$\mathrm{T}_{1}$ & $0,0043 \mathrm{a}$ & $0,0006 \mathrm{~b}$ & $0,095 \mathrm{a}$ & $0,015 \mathrm{a}$ & $0,009 \mathrm{a}$ \\
$\mathrm{T}_{2}$ & $0,0046 \mathrm{a}$ & $0,0011 \mathrm{~b}$ & $0,081 \mathrm{~b}$ & $0,015 \mathrm{a}$ & $0,010 \mathrm{a}$ \\
$\mathrm{T}_{3}$ & $0,0045 \mathrm{a}$ & $0,0011 \mathrm{~b}$ & $0,068 \mathrm{~b}$ & $0,012 \mathrm{a}$ & $0,010 \mathrm{a}$ \\
$\mathrm{T}_{4}$ & $0,0036 \mathrm{a}$ & $0,0019 \mathrm{a}$ & $0,109 \mathrm{a}$ & $0,016 \mathrm{a}$ & $0,013 \mathrm{a}$ \\
$\mathrm{T}_{5}$ & $0,0014 \mathrm{a}$ & $0,0006 \mathrm{~b}$ & $0,042 \mathrm{~b}$ & $0,007 \mathrm{a}$ & $0,005 \mathrm{a}$ \\
\hline $\mathrm{T}_{6}$ & & &
\end{tabular}

Letras distintas na mesma coluna diferem entre si pelo teste de Scott-Knott, ao nível de $5 \%$ de probabilidade.

\section{CRESCIMENTO DAS PLANTAS}

Os resultados referentes às características de crescimento das mudas de angelim-pedra, mostram que houve efeito dos tratamentos para todas as características avaliadas: altura $(\mathrm{F}=4,533 ; \mathrm{p}<0,05)$, diâmetro $(\mathrm{F}=6,657 ; \mathrm{p}<0,05)$, MSPA $(\mathrm{F}=2,667 ; \mathrm{p}<0,05), \operatorname{MSR}(\mathrm{F}=3,116 ; \mathrm{p}<0,05)$ e MST $(\mathrm{F}=2,767$; $\mathrm{p}<0,05)$, com exceção da relação raiz/parte aérea (RRPA), que foi estatisticamente igual entre os tratamentos aplicados (Figura 1; Tabela 5).

Os valores de altura e matéria seca da parte aérea (MSPA) das mudas tratadas com calcário dolomítico $\left(\mathrm{T}_{1}\right)$, calcário magnesiano $\left(\mathrm{T}_{2}\right)$, calcário calcítico $\left(\mathrm{T}_{3}\right)$ e aplicação de Ca e Mg na relaçáo 3:1 $\left(\mathrm{T}_{4}\right)$ e 9:1 $\left(\mathrm{T}_{5}\right)$ foram estatisticamente iguais e superiores ao tratamento com $\mathrm{Ca}$ e $\mathrm{Mg}$ na relaçáo 15:1 $\left(\mathrm{T}_{6}\right)$ e testemunha $\left(\mathrm{T}_{0}\right)$. Tais resultados sugerem que a calagem é importante para a produção de mudas desta espécie, no entanto, a correção da acidez do substrato pode não ser o principal benefício da calagem e sim a correção dos teores de $\mathrm{Ca}^{2+} \mathrm{e} \mathrm{Mg}^{2+}$ do solo, tendo em vista as respostas observadas nos tratamentos $\mathrm{T}_{2}$ e $\mathrm{T}_{5}$.

Em relação ao diâmetro, verificou-se que as mudas produzidas com calcário magnesiano $\left(\mathrm{T}_{2}\right)$ apresentaram um diâmetro estatisticamente superior ao observado nos demais tratamentos (Tabela 3). As mudas tratadas com calcário dolomítico $\left(\mathrm{T}_{1}\right)$, calcário calcítico $\left(\mathrm{T}_{3}\right)$, relação Ca: $\mathrm{Mg}$ de $3: 1$ $\left(T_{4}\right)$ e 9:1 $\left(T_{5}\right)$ não apresentaram diferenças significativas entre si, diferindo apenas dos tratamentos $\mathrm{T}_{6}$ (relaçáo Ca: $\mathrm{Mg}$ de 15:1) e $\mathrm{T}_{0}$ (testemunha), que foram estatisticamente iguais e com os menores resultados. Isso indica que, para esta variável, a utilização de um calcário com adequado teor de Ca e Mg é mais indicado que a adição de $\mathrm{Ca}$ e $\mathrm{Mg}$ ao solo, proveniente de fontes não corretivas da acidez. Esta resposta pode ser restrita ao angelim-pedra. Curti Júnior (2001) estudando o efeito da calagem para as espécies ingá (Inga marginata Willd.), orelhade-negro (Enterolobium contortisiliquum (Vell.) Hauman),

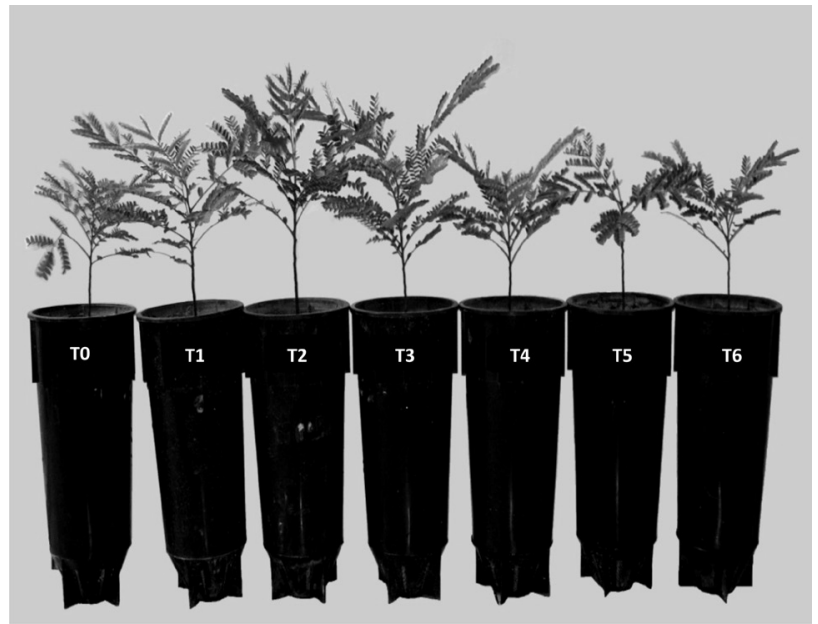

Figura 1 - Mudas de angelim-pedra (Dinizia excelsa Ducke) produzidas com diferentes calcários e doses de $\mathrm{Ca}$ e Mg. $\mathrm{T}_{0}$ - testemunha; $\mathrm{T}_{1}$ - calcário dolomítico; $T_{2}$ - calcário magnesiano; $T_{3}$ - calcário calcítico; $T_{4}$ - relação Ca:Mg de 3:1; $T_{5}$ - relação Ca:Mg de 9:1; e $\mathrm{T}_{6}$ - relação Ca:Mg de 15:1.

Tabela 5 - Variáveis mensuráveis das mudas de angelim-pedra produzidas na casa de vegetação da FCA, por um período de 152 dias.

\begin{tabular}{lcccccc}
\hline \multirow{2}{*}{ Tratamentos } & Altura & Diâmetro & MSPA & MSR & MST & RRPA \\
\cline { 2 - 7 } & $(\mathrm{cm})$ & $(\mathrm{mm})$ & \multicolumn{5}{c}{$\mathrm{g}$} & \\
\hline $\mathrm{T}_{0}$ & $14,82 \mathrm{~b}$ & $2,80 \mathrm{c}$ & $2,20 \mathrm{~b}$ & $0,83 \mathrm{~b}$ & $3,03 \mathrm{~b}$ & $0,39 \mathrm{a}$ \\
$\mathrm{T}_{1}$ & $17,08 \mathrm{a}$ & $3,41 \mathrm{~b}$ & $3,32 \mathrm{a}$ & $1,08 \mathrm{~b}$ & $4,39 \mathrm{a}$ & $0,33 \mathrm{a}$ \\
$\mathrm{T}_{2}$ & $19,56 \mathrm{a}$ & $3,84 \mathrm{a}$ & $3,98 \mathrm{a}$ & $1,56 \mathrm{a}$ & $5,54 \mathrm{a}$ & $0,40 \mathrm{a}$ \\
$\mathrm{T}_{3}$ & $18,11 \mathrm{a}$ & $3,28 \mathrm{~b}$ & $3,27 \mathrm{a}$ & $1,08 \mathrm{~b}$ & $4,35 \mathrm{a}$ & $0,35 \mathrm{a}$ \\
$\mathrm{T}_{4}$ & $17,25 \mathrm{a}$ & $3,17 \mathrm{~b}$ & $2,89 \mathrm{a}$ & $0,93 \mathrm{~b}$ & $3,83 \mathrm{~b}$ & $0,33 \mathrm{a}$ \\
$\mathrm{T}_{5}$ & $18,55 \mathrm{a}$ & $3,32 \mathrm{~b}$ & $3,79 \mathrm{a}$ & $1,11 \mathrm{~b}$ & $4,89 \mathrm{a}$ & $0,29 \mathrm{a}$ \\
$\mathrm{T}_{6}$ & $15,79 \mathrm{~b}$ & $2,66 \mathrm{c}$ & $1,99 \mathrm{~b}$ & $0,78 \mathrm{~b}$ & $2,77 \mathrm{~b}$ & $0,40 \mathrm{a}$ \\
\hline
\end{tabular}

Letras distintas na mesma coluna diferem entre si pelo teste de Scott-Knott, ao nível de $5 \%$ de probabilidade. MSPA - Matéria seca da parte aérea, MSR - matéria seca da raiz; MST - matéria seca total; RRPA - Relação raiz/Parte aérea.

angico-vermelho (Anadenanthera macrocarpa (Benth.) Brenan) e jacarandá-da-Bahia (Dalbergia nigra (Vell.) Allemao ex Benth.), verificou que apenas a orelha-de-negro apresentou efeito positivo à adição de calcário, mostrando que a resposta das espécies a adição da calagem depende das características intrínsecas de cada espécie.

Em relação à produçáo de matéria seca da raiz (MSR), as mudas de angelim-pedra tratadas com calcário magnesiano $\left(\mathrm{T}_{2}\right)$ apresentaram resultados significativamente superiores às produçōes médias observadas nos demais tratamentos, que se mostraram estatisticamente iguais (Tabela 3 ).

A produção de matéria seca total (MST) foi significativamente superior nas mudas tratadas com calcário dolomitico $\left(\mathrm{T}_{1}\right)$, calcário magnesiano $\left(\mathrm{T}_{2}\right)$, calcário calcítico $\left(\mathrm{T}_{3}\right)$ e aplicação de $\mathrm{Ca}$ e $\mathrm{Mg}$ na relaçấo 9:1 $\left(\mathrm{T}_{5}\right)$, sendo 
estatisticamente superiores as produçóes observadas nas mudas tratadas com a aplicação de Ca e $\mathrm{Mg}$ na relação 3:1 ( $\left.\mathrm{T}_{4}\right), 15: 1$ $\left(\mathrm{T}_{6}\right)$ e testemunha $\left(\mathrm{T}_{0}\right)$.

Não houve efeito dos tratamentos na relação raiz/parte aérea, isto é, não houve alteração na produção de matéria seca da raiz em relação a matéria seca da parte aérea. Esses resultados indicam que as plantas de angelim-pedra não desenvolveram o sistema radicular como mecanismo para compensar a ausência de corretivo ou Ca e Mg no solo (Tabela 3).

Houve diferença entre os diferentes tipos de calcários aplicados para as características de crescimento, sendo que as mudas tratadas com calcário magnesiano $\left(\mathrm{T}_{2}\right)$ apresentaram as maiores médias em relação à altura, diâmetro, MSPA, MSR e MST. As mudas tratadas com adição de $\mathrm{Ca}$ e $\mathrm{Mg}$ na relação 15:1 $\left(\mathrm{T}_{6}\right)$ e testemunha $\left(\mathrm{T}_{0}\right)$ foram as que apresentaram as menores médias (Tabela 3).

As mudas expostas aos tratamentos $\mathrm{T}_{2}$ e $\mathrm{T}_{5}$ apresentaram os maiores valores no peso da matéria seca da raiz (MSR), apesar de apresentarem diferença significativa entre si, o que estaria afetando de forma positiva todas as demais características de crescimento da espécie. Isso porque com um sistema radicular bem desenvolvido a absorção dos nutrientes pode ser mais eficiente, não sendo afetada de forma negativa pela acidez do substrato (Zonta et al. 2006).

Considerando apenas as relaçóes Ca:Mg testadas, observou-se que os maiores valores obtidos foram sob a relação 9:1 $\left(\mathrm{T}_{5}\right)$, correspondendo à relação $\mathrm{Ca:Mg}$ do calcário magnesiano. Semelhantemente, Furtini Neto et al. (1999) comparando o efeito da calagem, adição de $\mathrm{Ca}, \mathrm{Mg}$ e $\mathrm{OH}$ no substrato de cultivo de cássia verrugosa (Senna multijuga), ipê mirim (Stenolobium stans), angico-do-cerrado (Anadenanhera falcata) e cedro (Cedrela fissilis), observaram que mesmo sob condiçóes de acidez moderada o fornecimento de $\mathrm{Ca}$ ou $\mathrm{Mg}$ favoreceu o crescimento da maioria das espécies, mostrando efeito comparável ao da calagem, verificando que níveis adequados de cálcio ou magnésio podem amenizar os problemas relacionados à toxidez de alumínio, contudo, o efeito observado nas plantas pelo suprimento de $\mathrm{Ca}$ ou $\mathrm{Mg}$ pode diferir em função de características intrínsecas às espécies e/ou do tipo de solo.

Souza (2006), estudando diferentes relaçóes Ca:Mg do corretivo sobre o crescimento e qualidade das mudas de bico-de-pato (Machaerium nictitans (Vell.) Benth.), paujacaré (Piptadenia gonoacantha (Mart.) Macbr.) e fedegoso (Senna macranthera (Collad.) Irwin et Barn.) produzidas em diferentes tipos de solo, verificou que para as mudas de bicode-pato a melhor relação Ca: $\mathrm{Mg}$ do corretivo em Argissolo e Latossolo álico foi de 4:1 e no Latossolo distrófico foi de 2:1. Para as mudas de pau-jacaré a relaçáo Ca:Mg do corretivo, que forneceu as melhores mudas foi de 1:1 no Argissolo, 4:1 no Latossolo distrófico e 2:1 no Latossolo álico. Para as mudas de fedegoso, a melhor relação $\mathrm{Ca}: \mathrm{Mg}$ do corretivo, para os três tipos de solo, para as três espécie estudadas foi de 3:1. Isso mostra que o desempenho das espécies ainda pode ser influenciado pelo tipo de solo.

As mudas de angelim-pedra tratadas com calcário magnesiano $\left(\mathrm{T}_{2}\right)$ foram as que apresentaram os maiores valores de crescimento relativo em altura e diâmetro, com aumento de $34,3 \%$ e $37,7 \%$, respectivamente, em relação a testemunha $\left(\mathrm{T}_{0}\right)$. As mudas tratadas sem adição de calagem ou Ca e $\mathrm{Mg}\left(\mathrm{T}_{0}\right)$ foram as que apresentaram o menor crescimento relativo em altura e as mudas tratadas com adição de $\mathrm{Ca}$ e $\mathrm{Mg}$ na relação 15:1 $\left(\mathrm{T}_{6}\right)$ foram as que apresentaram o menor crescimento relativo em diâmetro, com um decréscimo de $4,6 \%$, em relação a testemunha $\left(\mathrm{T}_{0}\right)$ (Figura 2$)$.

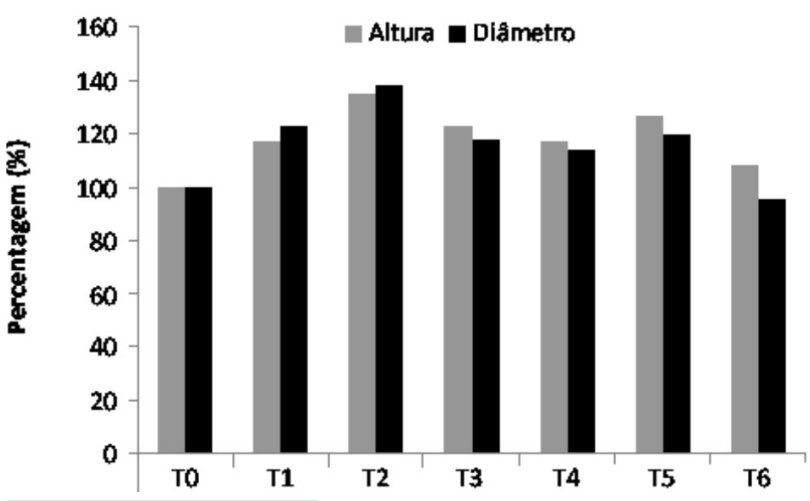

Figura 2 - Crescimento relativo em altura e diâmetro de mudas de angelimpedra produzidas em casa de vegetação por um período de 152 dias.

Em relação ao crescimento relativo em MSPA, MSR e MST, verificou-se que as mudas tratadas com calcário magnesiano $\left(\mathrm{T}_{2}\right)$ apresentaram os maiores valores de crescimento relativo, observando-se um crescimento de $84,9 \%, 83,8 \%$ e $83,4 \%$, respectivamente, comparados à testemunha $\left(\mathrm{T}_{0}\right)$ (Figura 3). As mudas tratadas com adiçáo de $\mathrm{Ca}$ e $\mathrm{Mg}$ na relação 15:1 ( $\mathrm{T}_{6}$ ) foram as que apresentaram o menor crescimento relativo em MSPA, MSR e MST, com um decréscimo de 6,7\%, 5,09\% e 6,96\%, respectivamente, em relação à testemunha $\left(\mathrm{T}_{0}\right)$, tais resultados indicam que um desequilíbrio na relação Ca: $\mathrm{Mg}$ pode provocar prejuízos no desenvolvimento de mudas desta espécie.

Observa-se também que os maiores valores para todas as características avaliadas foram obtidos mediante o tratamento com calcário magnesiano $\left(\mathrm{T}_{2}\right)$, indicando que esta espécie desenvolve-se melhor quando o substrato de cultivo for corrigido com produtos que tenham uma relação Ca: $\mathrm{Mg}$ de 9:1, conforme observado quando houve aplicação de Ca e $\mathrm{Mg}$, provenientes de fontes náo corretivas da acidez.

Verificou-se que náo houve diferença significativa entre a produção de matéria seca total (MST) das mudas submetidas 


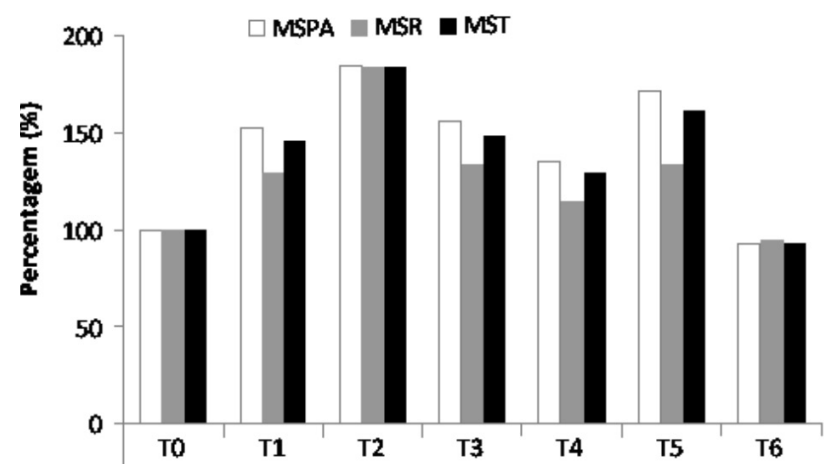

Figura 3 - Crescimento relativo em matéria seca da parte aérea (MSPA), matéria seca da raíz (MSR) e matéria seca total (MST) de mudas de angelimpedra produzidas em casa de vegetação por um período de 152 dias.

à calagem $\left(\mathrm{T}_{1}, \mathrm{~T}_{2}\right.$ e $\left.\mathrm{T}_{3}\right)$ e a adição de $\mathrm{Ca}$ e $\mathrm{Mg}$ na relaçáo 9:1 $\left(\mathrm{T}_{5}\right)$. Sendo que as plantas submetidas a adição de $\mathrm{Ca}$ e $\mathrm{Mg}$ na relação 3:1 $\left(\mathrm{T}_{4}\right)$, 15:1 $\left(\mathrm{T}_{6}\right)$ e testemunha $\left(\mathrm{T}_{0}\right)$ foram iguais.

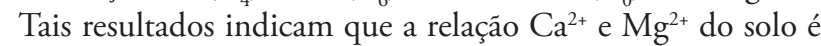
importante para o desenvolvimento desta espécie, quando a correção da acidez do substrato não for realizada.

Aparentemente, o angelim-pedra é uma espécie tolerante a acidez moderada do solo e a maior produção de matéria seca total (MST) foi obtida nos tratamento onde a relação Ca e $\mathrm{Mg}$ adicionada ao substrato foi de 9:1, tanto para a calagem $\left(\mathrm{T}_{2}\right)$ quanto para o produto náo corretivo da acidez $\left(\mathrm{T}_{5}\right)$.

\section{CONCLUSÕES}

O calcário magnesiano e a aplicação de $\mathrm{Ca}$ e $\mathrm{Mg}$ na relação de 9:1, oriundos de fontes não corretivas da acidez, foram os tratamentos mais indicados para a produção de mudas de angelim-pedra (Dinizia excelsa Ducke), por promoverem maior crescimento e maior absorção de nutrientes.

\section{BIBLIOGRAFIA CITADA}

Barros, J.G. 2001. Liming and fertilization to the formation of seedlings of mogno (Swietenia macrophylla King). Dissertação de Mestrado, Universidade Federal do Amazonas, Manaus, Amazonas. 64pp (in Portuguese).

CFSEMG. 1999. Recommendations for the use of fertilizers and in Minas Gerais. 5a. Aprox. Viçosa, Minas Gerais. 359pp (in Portuguese).

Curti Júnior, H.M. 2001. Effect of aluminum and calcium in the growth ofleguminous trees. Dissertação de Mestrado, Universidade Federal Rural do Rio de Janeiro, Seropédica, Rio de Janeiro. 100pp (in Portuguese).

EMBRAPA. 1997. Manual methods of soil analysis. $2^{\text {a }}$ ed. Rio de Janeiro, Centro Nacional de Pesquisa de Solos. 212pp (in Portuguese).

Fearnside, P.M. 1998. Agro-forestry policy in development policy in the Brasilian Amazon: the importance and the limits of its use in degraded areas, p. 293-312. In: Gascon, C.; Moutinho, P. (eds), Rainforest: dynamic regeneration and management. Instituto Nacional de Pesquisa da Amazônia, Manaus, Amazonas (in Portuguese).

Furtini Neto, A.E.; Resende, A.V.; Faquin, V.; Vale, F.R.; Fernandes, L.A. 1999. Soil acidity, growth and mineral nutrition of some tree species at seedling phase. Cerne, 5(2): 1-12 (in Portuguese, with abstract in English).

Gascon, C.; Moutinho, P. 1998. Síntese, p. 333-337. In: Gascon, C .; Moutinho, P. (eds). Rainforest: dynamic regeneration and management. Instituto Nacional de Pesquisas da Amazônia, Manaus, Amazonas (in Portuguese).

Gomes, F.T.; Borges, A.C.;Neves, J.C.L.; Fontes, P.C.R. 2002. Influence of limestone rates with different calcium:magnesium ratios on shoot dry matter yield and mineral composition of alfalfa. Pesq. Agropec. Bras. 37(12): 1779-1786 (in Portuguese, with abstract in English).

Malavolta, E.; Vitti, G.C.; Oliveira, S.A. 1997. Assessment of nutritional status of plants: principles and applications. $2^{\text {a }}$. ed. Potafos, Piracicaba. 319pp (in Portuguese).

Paiva, H.N.; Gomes, J.M. 2000. Nurseries. 2a. ed. Universidade Federal de Viçosa, Viçosa, Minas Gerais (Cadernos didáticos). 69pp (in Portuguese).

Quaggio, J.A. 2000. Acidity and liming in tropical soils. Instituto Agronômico de Campinas, Campinas, São Paulo. 111pp (in Portuguese).

Rossielo. R.O.P.; Jacob Netto, J. 2006. Aluminum toxicity in plants: new approaches to an old problem, p.375-418. In: Fernandes, M.S. (ed), Mineral nutrition of plants. Sociedade Brasileira de Ciência do Solo, Viçosa, Minas Gerais (in Portuguese).

Silva, A.R.M. 2004. Liming for the production of seedlings of mogno (Swietenia macrophylla King.) and cedro (Ceiba pentandra L. Gaertn). Dissertação de Mestrado, Universidade Federal do Amazonas, Manaus, Amazonas. 53pp (in Portuguese).

Silva, A.R.M. Tucci, C.A.F.; Lima, H.N.;Figueiredo, A.F. 2007. Growing doses of liming on mogno (Swietenia macrophylla King) in seedling formation. Acta Amazonica, 37(2): 195-200 (in Portuguese, with abstract in English).

Silva Júnior, C.H. 2006. Responses to fertilization with nitrogen, phosphorus and potassium in the formation of seedlings of mogno (Swietenia macrophylla King.) and cedro (Ceiba pentandra L. Gaertn). Dissertação de Mestrado, Universidade Federal do Amazonas, Manaus, Amazonas. 56pp (in Portuguese).

Souza, P.H. 2006. Growth and quality of seedlings pau de jacaré (Piptadenia gonoacantha (Mart.) Macbr.), bico de pato (Machaerium nictitans (Vell.) Benth.) and fedegoso (Senna macranthera (Collad.) Irwin et Barn.) in response to liming. Dissertação de Mestrado. Universidade Federal de Viçosa, Viçosa, Minas Gerais. 62pp (in Portuguese).

Uhl, C.; Buschbacher, R.; Serrão, E.A.S. 1988. Abandoned pastures in easterb Amazonian. I. Patterns of plant succession. Journal of Ecology. 76:663-681.

Vastano, J.R.B.; Barbosa, A.P.; Gonçalves, A.N. 1983. Pre-germinative treatments in seeds of species of Amazonian forest seeds. I. Angelim- 
stone (Dinizia excelsa Ducke, Leguminosae-Mimosoideae). Acta Amazonica, 13(2): 413-419 (in Portuguese, with abstract in English).

Venturini, R.P.; Bastos, A.R.R.; Mendonça, A.V.R.; Carvalho, J.G. de. 2000. Effects of the Ca:Mg relation on the development and mineral nutrition of aroeira plants (Myracrodruon urundeuva Fr. All.). Cerne, 6(1): 030-039 (in Portuguese, with abstract in English).

Veloso, C.A.C.; Souza, F.R.S.; Pereira, W.L.M.; Tenório, A.R.M. 2001. Effect of calcium, magnesium, and potassium rate of dray matter of corn. Acta Amazônica, 31(2): 193-204 (in Portuguese, with abstract in English).

Vitti, G.C.; Lima, E.; Cicarone, F. 2006. Calcium, Magnesium and Sulfur. In: Fernandes, M.S. (ed). Nutrição mineral de plantas. Sociedade Brasileira de Ciência do Solo, Viçosa, Minas Gerais, p.299-325 (in Portuguese).
Ulhôa, M.L. 1997. Effect of liming and fertilization on the growth and nutrition of plants baru (Dipteryx alata Vog.) fruto-de-lobo (Solanum lycocarpum St. Hil) and tingui (Magonia pubescens St. Hil). Dissertaçáo de Mestrado. Universidade Federal de Lavras, Lavras, Minas Gerais. 74pp (in Portuguese).

Zonta, E.; Brasil, F. da C.; Goi, S.R., Rosa, M.M.T. da. 2006. The root system and its interactions with the soil environment, p.07-52. In: Fernandes, M.S. (ed). Mineral nutrition of plants. Sociedade Brasileira de Ciência do Solo, Viçosa, Minas Gerais (in Portuguese).

Recebido em: 04/02/2009

Aceito em: 11/11/2009 
